\title{
Analysis on Brushwork of Chinese Watercolor Painting
}

\author{
Qiaoqiao Wang \\ Shaanxi Normal University \\ Shaanxi, China
}

\begin{abstract}
In the process of Chinese watercolor painting development, Chinese watercolor artists follow western painting methods and use traditional Chinese painting's brushwork for reference at the same time, emphasizing brushwork's sense of potency and freehand character. They naturally blend aesthetic consciousness of Chinese painting's brushwork in watercolor painting and greatly expand watercolor painting's expressional language. This paper takes traditional brushwork of ink and wash painting as the entry point to discuss important significance and realistic value of pursuit of brushwork on the road of watercolor's nationalization.
\end{abstract}

Keywords—brushwork; aesthetic interest; brushwork structure

\section{ReSEARCh Traditions AND MaKe the PASt SERVE THE PRESENT}

The ancients value brushwork in terms of painting. There are a lot of discussions about brushwork in history. Brushwork is a category which can display national style the most in Chinese fine arts theory. It not only involves tool materials used in creation activity. More importantly, it displays beauty in form. In Qi and Liang period, art theorist Xie He's "six methods of painting" summarizes a series of basic requirements of Chinese creation of painting. "Bone method of brushwork" comes after "lifelikeness" and is listed as the second important method of painting, proving its important status in painting. In fact, connotation of "brushwork" is very rich and it has the direct bearing on expression of inherent spirit of artwork. In Tang dynasty, Zhang Yanyuan says "Object resembling lies in similarity in form which needs bone spirit. Bone spirit and similarity in form are based on conception and they return to brushwork. Therefore most painters are good at calligraphy." (1) In Northern Song, Guo Ruoxu says:" Artistic conception of painting concerns mind excursion. Vividness comes from brushwork. Therefore it is clear that brushwork is difficult." (2) In China's artistic concept, "bone spirit", "conception", "artistic conception" and "vividness" are the highest state expressed by artwork and they are closely related to brushwork. Whether can we get some enlightenment from these works, summarize common artistic laws and apply them in artistic creation of watercolor painting? Artistic laws are interlinked. As to Chinese watercolor painters expecting to expand a nationalized watercolor road, I think it is available to start with research on tradition.

\section{UNDERSTANDING OF BRUSHWORK IN WATERCOLOR PAINTING FROM TRADITIONAL BRUSHWORK METHOD}

Ancient people have a deep understanding of brushwork. As to how to do brushwork, they put forward many theories with features, interest and charm. Here are several examples. "As to brushwork, every brushstroke should be solid with emptiness. Emptiness brings about spirituality without hysteresis. Without hysteresis, the work is totally mixed up in wonderfulness, making it a heavenly creation." "Although brushwork comes to an end, the connotation is boundless. It is called emptiness." "Emptiness can be seen in ancients' solid brushwork." Qi Baishi also says: "Artful and clumsy brushwork is needed in landscape painting. Artful brushwork produces spirituality and clumsy brushwork produces simple and vigorous sense. It is in accordance with heaven, creation of heaven naturally has no frivolous or muddy weakness." A mature painter's selection and preference of brushwork style not only reflects his comprehensive quality and temperament peculiarity. The shine of his personality and moral character of literators is also reflected in the overall light beam of his language series. I will discuss them separately in the following part.

\section{A. Brushwork and Emotion}

As the main body of traditional artistic language, brushwork style is psychological scanning of human's conversation with nature. Various different elegant demeanour and brushwork with different characters are various feelings aroused in people's mind by various states in the objective world. Painters do painting out of feeling and brushwork style is imitation and memoir of this feeling.

"Breaking away from life, brushwork can't be magical." Li Yu can produce "Ask the gentleman how much he worries", because he has more worries than others. That's why his tears are like river water. It is exactly when his feeling is deeper than others', his work can be moving. Li Yu's figure of speech is different from his father's. His father is Li Jing who writes "Cloves knot melancholy in rain". People who have seen cloves know the look of clove. But very few people observe cloves in rain. There is a drop of tear on every petal. With different descriptions, the same melancholy and tear can be so vivid. Brushwork can't be great without life or real feelings in life. 
"Process of pen movement" refers to interaction between pen and link and paper and the whole process of leaving traces on Chinese art paper. Pen movement traces are left in process of drawing, including ink lines. Seeing from ink trace storage process, line's movement is the most complex and it falls into three steps: first stroke, wielding the pen and end stroke. First stroke is the beginning of line, wielding the pen is running of line, and end stroke means termination of line. Chinese painting's pen wielding has the same source with that of calligraphy. In Yuan dynasty, Zhao Mengtiao writes:" Brushwork of cursive script is used in stone drawing, brushwork of seal script is used in wood drawing, and eight methods of "Yong" are used in writings about bamboo. If there are people knowing about relation between painting and calligraphy, they must know that calligraphy and painting are homogenous in brushwork."(3) Calligraphy emphasizes correct writing methods and cooperative application of elbow and wrist. As to pen wielding methods, there is silver shadow, versa painting, brush-side technique, brush-center technique and so on. Chinese painting is also the same. In previous dynasties, many traditional Chinese painters are good at calligraphy and they bring calligraphy into painting, forming various characteristic painting style and features. Here are several examples. Xu Wei uses cursive script and Zheng Banqiao uses cursive characters. Shi Tao says "Ancients combine eight methods into six methods and they use calligraphy in painting, like Zhuan, Cao and Li".(4) When mentioning brushwork, traditional painting and calligraphy especially emphasize "completion at one stroke with qi channel cut-through," which is "lifelikeness" in six methods. What does "artistic conception" here refer to? I think it mainly refers to painter's feeling, emotion and state of mind which run through brushwork in painting. Watercolor painting and traditional calligraphy and painting are similar in brushwork. In watercolor painting creation, stroke's lifting, pressing, pausing, lowering, picking and clicking are either strong or weak, either slow or quick, either open or restrained, either static or dynamic, either ingenious or upright, and either complex or simple. Brushwork moves with emotion and state of mind, and provides subjective expression with great possibilities and a wide world.

From Mr Wang Zhaomin's repeated and simple brushwork, we can feel his simplicity, effort, and extremely vigorous and smooth lofty and unyielding vigour. From $\mathrm{Mr}$ Chen Shaoping's speedy and smooth brushwork, we can taste a kind of relaxing, sprightly, tranquil and forthright and sincere romantic charm. Mr Wang Weixin displays spirituality and unrestrained character in courage and liberty. Mr Lei Hong displays ethereal and unconstraint in rolling and flying. It can be said that brushwork of Chinese watercolor painting with strong infection is definitely sympathia, unison, collision and fusion of painter's internal vitality pulsation and external vitality and vigor of nature. Watercolor painting with calligraphy and calligraphic beauty definitely is national in style.

\section{B. Brushwork and Content}

Painters do painting out of feelings and experiences in life. "Painters should regard nature as their teacher and combine with inner feelings to create excellent works.” Before painting, painters need to observe external modeling of object and extract and purify to the artistic level. Therefore, painting process isn't purely depiction and reproduction of object. It is a process of self vent and self expression through reproduction of image. So we can't ignore internal feelings about objects. For example, "modeling features and peculiar texture". Content determines form. There is a kind of corresponding pattern of manifestation on speaking terms with a kind of content. Different contents have different patterns of manifestation and different techniques of expression.

Take landscape painting in Chinese traditional painting as an example, traditional wrinkle method leaves us precious wealth. There is ox hair wrinkle for ox hair, spot wrinkle for sesame, big and small stroke of axe for hard massifs, ghostface wrinkle for karst landform and so on. These brushwork methods are formed through predecessors' creation. Predecessors have so many classifications for a single wrinkle method, because of different hillstone structures and different manifestation textures.

Brushwork's excellence lies in meaning. Brushwork stresses that "In horizontal drawing, there should be an end stroke of left "turning". In vertical drawing, there should be shrinkage at the tail end of stroke." It is pursuit of mellow brushwork under guidance of meaning. Brushwork of catfish in Badashanren's painting is unrestrained by "silver shadow" and "twists and turns". Generally floating brushwork without silver shadow is regarded as a failure. But in this painting, it endows the image with a skittery feeling, having a fantastic effect. Another example is $\mathrm{Mr}$ Tao Shihu drawing snowcovered landscape. He likes presenting tranquil, broad and profound scenes. He also likes natural flow of emotion in ethereal and heaviness. Therefore, his artistic orientation determines his technique of expression in painting. In the painting Yimeng Snow, we can clearly see his application of dry brush "hollow stroke" blank leaving over-dye technique. He boldly imitates Chinese painting's brushwork techniques and uses traditional "bone method" to the top of his bent. There is wrinkling, rubbing, outline drawing, and strong, weak, fast and slow stroke. He also uses disperse dry brush's twists and turns and jerking changes of rise and down to display texture of trees, grass and hillstone. There is a large area of snowfield in the painting. Handling of snowfield definitely is particular about techniques. We can see that his brushwork develops with object's structure. Seeing from surface, it seems that there is no exposed brushwork. With scrutiny, every stroke is vivid and full of springiness. There is no sense of complexity in various strokes. The whole painting shows great beauty of calligraphic style with looseness and impressiveness. Therefore, the key of brushwork is serving the content and painter's purpose, and it should be tenable. I think these principles can also be applied in watercolor painting.

\section{Brushwork and Texture}

In painting, painter's application of different brushwork leads to different styles of drawing. The brushwork can be light, strong, smooth or vast, and it leaves different traces on paper with rich performance. This kind of performance is transferred to audience visually, thus forming a certain kind of 
texture. Uncertainty of artistic image usually is the precondition to create infinity of aesthetic state. Form beauty of texture itself has relatively independent aesthetic significance.

Today, with diversified artistic techniques of expression, many painters are passionate about try of different textures. They think that "Various texture phenomena relate to human's psychological effect. If various texture patterns are regarded as a kind of visual information which is transformed into painting language to convey feelings and ideas. Undoubtedly, it will enrich and enhance painting art's expressive force." This understanding is correct with certain positive significance. But what makes me doubtful is what the function of creation subject's subjective effort is at the same time with their using of various textures to improve watercolor's artistic expressive force. Whether unreasonable texture manifestation leads to loss of watercolor language's independence? These questions are worth discussing.

My saying is not rejection of relying on certain textures to deal with painting in watercolor painting creation. For example: When creating the landscape painting of Guan Mountain Series, Mr Lv Zhikai's dealing with grassland is worth our learning. In creating grassland, he first uses a spray gun to rinse out surface dyeing. Thus, the place washed by spray gun has a kind of natural effect due to water washing. After the painting is completely dry, dry brush "hollow stroke" blank leaving over-dye is applied to replenish and adjust, forming a kind of natural texture. It looks vigorous, soft, luxuriant, rich and natural with a strong sense of grassland. Obviously, in dealing with grassland, the painter just takes advantages of topography and makes a little use of texture in reserve after washing, then artistic purpose is achieved, just like "When conditions are ripe, success will come". A few strokes are better than thousands and thousands of strokes, obtaining the artistic effect of "Absence of stroke prevails over existence of stroke".

With artistic view becoming wider and wider and diversity in form language, watercolor art itself should keep distance with other artistic forms and it shouldn't deviate from independence of watercolor artistic language. Exploration of artistic language should pay attention to "heat control" and grasp a proper "degree". The key should be improvement of watercolor artistic quality. Thus, there is a bottom line in grasp of watercolor language. On this basis, artistic pattern of manifestation can be free. I think the best texture is brushwork, which records creation subject's emotional changes in painting process and bears painter's emotion. Natural texture formed by good brushwork can make people have random thoughts, leave a strong impression on people and move people.

\section{WATERCOLOR'S BRUSHWORK STRUCTURE}

As the ontology language of watercolor, brushwork structure occupies an important status and it is one of the most important signs of watercolor painting's technical content. Watercolor painting's brushwork emphasizes three points: accuracy, decisiveness and management. The so-called accurate brushwork refers to meaning coming before brushwork. With one stroke, water content, color and time difference are just exactly appropriate, and painting effect reaches painter's satisfactory degree. This is a link which is the most difficult to grasp in watercolor brushwork structure and it is the key of watercolor brushwork structure, where lies the secret of watercolor's lasting appeal. Decisiveness refers to that the painter can't hesitate or can't be indecisive and sloppy in brushwork. Indecisive brushwork of watercolor painting leads to loose, weak and feeble painting, lacking manifestation strength and impressiveness. As to decisiveness of brushwork, Guangdong painters are worth learning. Most of them inherit Wang Zhaomin's dry, rough and vigorous brushwork in painting with tension in painting. The third point is "management", which refers to conscious organization of painting. In many excellent painters' paintings, it seems that their brushwork is at will. In fact, it is the result of elaboration. For example, Ms. Gong Yu's watercolor painting of Tea House Series contains freedom and vividness with sense of "freehand brushwork" in Chinese painting. Bold and unrestrained brushwork is interlocked and completed at one stretch without loss in modeling. She skillfully uses images to organize brushwork and endows the painting with natural and strong rhythm, like "scrawling" brushwork moving about freely and quickly on paper. Learning from the nature and obtaining the will of heaven. This kind of artistic state can only be expected rather than obtained through seeking. With careful appreciation, you can find rigorous painting structure and particular brushwork in painting. Obviously, they are the result of elaboration. Accuracy, decisiveness and management make up three main aspects of watercolor's brushwork structure. These three aspects are independent outside the painting and unified in the painting. Just like a triangle having stability, only by close combination of those three aspects in brushwork, can a stable and reliable painting with expressive force and infectivity be produced. It seems that watercolor's brushwork is simple. But, to achieve systematic grasp of it to a high degree of professional proficiency, painters who are persistent in watercolor need to try to figure out attentively and experience deeply.

\section{CONCLUSION}

At the same time of learning and researching western artistic essence, contemporary Chinese watercolor painting has to firmly lay the roots in national soil and absorb its rich nutrition and quintessence. As an important constituent part of watercolor painting's ontology language, brushwork has important value and it is worth our constant imitation, inheriting, exploration and development of tradition. We have no reason to be used to feel at ease about watercolor painting's absence of brushwork, pallor and weakness with one-sided, single and stuffless aesthetic consciousness. No matter it is reproduction, manifestation, abstraction or concretization, what's important is expression of creation subject's emotion and spirit. "There is spirit in brushwork." As long as there is national emotion and spirit, the watercolor painting must have a style with national characteristics, thus having dazzling shine among world's watercolor paintings. 


\section{REFERENCES}

[1] Zhang Yanyuan. Annotation by Yu Jian. Famous Paintings of History[M]. Shanghai: Shanghai People's Fine Arts Press. 1964:13.

[2] Zhou Jiyin. Chinese Painting Theory Digest[M]. Nanjing: Jiangsu Fine Arts Press. 2005:461.

[3] Yu Jianhua. Chinese Ancient Art Theory Classified Compilation[M]. Beijing: People's Fine Arts Publishing House. 2004:1069.

[4] Yi Canghai. Brushwork with Rules and without Rules[J]. Tianjin Fine Arts Institute Journal. 2011(3):62. 